Meta

Journal des traducteurs

Translators' Journal

\title{
Les bases épistémologiques et linguistiques de la nomenclature chimique de 1787
}

\section{Henri Cottez}

Volume 39, numéro 4, décembre 1994

Hommage à Bernard Quemada : termes et textes

URI : https://id.erudit.org/iderudit/002408ar

DOI : https://doi.org/10.7202/002408ar

Aller au sommaire du numéro

Éditeur(s)

Les Presses de l'Université de Montréal

ISSN

0026-0452 (imprimé)

1492-1421 (numérique)

Découvrir la revue

Citer cet article

Cottez, H. (1994). Les bases épistémologiques et linguistiques de la nomenclature chimique de 1787. Meta, 39(4), 676-691.

https://doi.org/10.7202/002408ar
Résumé de l'article

Cette étude vise à mettre en évidence les bases épistémologiques et linguistiques de la nomenclature chimique de 1787, en partant des Mémoires présentés à l'Académie des Sciences par Lavoisier, Morveau, Fourcroy et Berthollet et du Traité de Chimie de Lavoisier (1789). Ces textes sont l'aboutissement d'une réflexion collective et pluridisciplinaire, dans la ligne de la philosophie de Condillac, qui fait d'une « langue bien faite » la condition d'une science véritable. Il ne s'agit donc pas d'une simple nomenclature nouvelle, mais d'une " méthode de nommer ». Un principe général est que les noms doivent être motivés, laissant transparaître la nature et la composition des corps chimiques (parallélisme entre le plan de l'expression et le plan du contenu). La suffixation, en rassemblant sous tel ou tel suffixe les " corps congénères " permettra d'identifier et de classer les substances découvertes et à découvrir. Accessoirement, on aura recours aux structures lexicales, particulièrement riches et maniables, de l'ancien grec. Enfin, pour maintenir le parallélisme dans les cas difficiles (combinaisons multiples d'éléments), les réformateurs vont réduire des unités lexicales à un court morphème chargé de les représenter, comme pyro pour empyreumatique et hydr(o)-pour hydrogène . C'est ce que l'auteur a appelé la " fonction de représentation », qui est aujourd'hui à l'œuvre dans tout le vocabulaire savant.
Ce document est protégé par la loi sur le droit d'auteur. L'utilisation des services d'Érudit (y compris la reproduction) est assujettie à sa politique d'utilisation que vous pouvez consulter en ligne.

https://apropos.erudit.org/fr/usagers/politique-dutilisation/ 


\title{
LES BASES ÉPISTÉMOLOGIQUES ET LINGUISTIQUES DE LA NOMENCLATURE CHIMIQUE DE 1787
}

HENRI COTTEZ

Paris, France

\begin{abstract}
Résumé
Cette étude vise à mettre en évidence les bases épistémologiques et linguistiques de la nomenclature chimique de 1787, en pärtant des Mémoires présentés à l'Académie des Sciences par Lavoisier, Morveau, Fourcroy et Berthollet et du Traité de Chimie de Lavoisier (1789). Ces textes sont l'aboutissement d'une réflexion collective et pluridisciplinaire, dans la ligne de la philosophie de Condillac, qui fait d'une "langue bien faite» la condition d' une science véritable. Il ne s'agit donc pas d'une simple nomenclature nouvelle, mais d'une «méthode de nommer». Un principe général est que les noms doivent être motivés, laissant transparaître la nature et la composition des corps chimiques (parallélisme entre le plan de l'expression et le plan du contenu). La suffixation, en rassemblant sous tel ou tel suffixe les "corps congénères» permettra d'identifier et de classer les substances découvertes et à découvrir: Accessoirement, on aura recours aux structures lexicales, particulièrement riches et maniables, de l'ancien grec. Enfin, pour maintenir le parallélisme dans les cas difficiles (combinaisons multiples d'éléments), les réformateurs vont réduire des unités lexicales à un court morphème chargé de les représenter, comme pyro pour empyreumatique et hydr(o)pour hydrogène. C'est ce que l'auteur a appelé la "fonction de représentation", qui est aujourd' hui à l'ouvre dans tout le vocabulaire savant.
\end{abstract}

\begin{abstract}
This study aims at exploring the epistemological and linguistic bases of the 1787 chemical nomenclature, using the Mémoires presented to the Académie des Sciences by Lavoisier, Morveaux, Fourcroy and Berthollet and the Traité de Chimie by Lavoisier (1789). These texts are the outcome of collective and pluridisciplinary thought along the lines laid out in the philosophy of Condillac, who maintained that a "well made language" was the prerequisite for any true science. The aim was thus not simply to create a new nomenclature, but to lay out a method for naming. One general principle was that names must be motivated, so that the nature and composition of chemical substances are made clear (i.e. a parallel between expression and content). The suffixation system used, grouping "congeneric substances" under the one suffix, make it possible to identify and to classify substances known and yet to be discovered. Ancient Greek lexical structures, particularly rich and flexible, were also put to use. Finally, to maintain motivation in difficult cases (multiple combinations of elements), the reformers decided to reduce lexical units to one short representative morpheme, such as pyro for empyreumatique and hydr(o) for hydrogène. This is what the author called "fonction de représentation", which is at work today in all scientific vocabulary.
\end{abstract}

En 1787 , au terme d'un travail collectif de plusieurs années visant à «réformer et perfectionner la nomenclature de la Chimie», les principaux artisans de cette audacieuse entreprise présentent, devant l'Académie des Sciences, un plan soigneusement élaboré, aussi précis que complet, qui fera date dans l'histoire de la chimie, et des sciences en général, dans la mesure où il est un modèle, jusqu'ici inégalé, de néologie scientifique. Les auteurs de cette véritable révolution sont quatre chimistes de grand renom, d'ailleurs

Meta, XXXIX, 4, 1994 
membres de cette Académie (à l'exception du provincial Guyton de Morveau), expérimentateurs et théoriciens à la fois : ils savent qu'ils risquent de rencontrer chez leurs confrères réticences ou résistances, mais ils ont assez fait leurs preuves pour qu'on doive les prendre au sérieux.

C'est Lavoisier, le plus célèbre, qui ouvre le feu, le 18 avril, en lisant son Mémoire sur la nécessité de réformer et de perfectionner la Nomenclature de la Chimie. Le 2 mai, Guyton de Morveau (qui avait été l'initiateur de ce grand projet en publiant une première esquisse dans le Journal de Physique de mai 1782), prend le relais avec un Mémoire sur le développement des principes de la Nomenclature méthodique, accompagné d'un Appendice contenant la Nomenclature de quelques substances composées qui se combinent quelquefois à la manière des corps simples. Suivra un troisième exposé, dû à Fourcroy, Mémoire pour servir à l'explication du Tableau de Nomenclature. Enfin, après un Avertissement sur les deux synonymies, les Académiciens seront en possession de la Synonymie ancienne et nouvelle par ordre alphabétique (Noms anciens-Noms nouveaux) et du Dictionnaire pour la nouvelle Nomenclature chimique (Noms nouveaux-Noms anciens, s'il y en a). Si Berthollet n'a pas été chargé d'un exposé particulier, il n'en est pas moins cité plusieurs fois comme un des quatre auteurs de la réforme. Notons que les Académiciens réagiront dès le 13 juin par un Rapport sur la nouvelle Nomenclature (sur lequel je reviendrai), signé Baumé, Cadet, Darcet et Sage, et certifié conforme par Condorcet.

À ces textes, tous importants à divers titres et profondément novateurs, il convient d'ajouter l'ouvrage capital publié en 1789 par Lavoisier: Traité élémentaire de Chimie, présenté dans un ordre nouveau et d'après les découvertes modernes. Car non seulement le grand chimiste revient, dans un magistral Discours Préliminaire, sur l'esprit et les principes de la réforme de 1787 , mais aussi, dans nombre de pages, comme on verra, il mêle à l'exposé scientifique des réflexions d'ordre plus général, qui éclairent d'un jour nouveau une démarche intellectuelle. L'édition de 1789 était suivie la même année d'une «Nouvelle édition», qui n'avait de nouveau que d'ajouter aux deux volumes de l'originale un troisième volume, précieux en ce qu'il renferme tous les Mémoires de 1787 . Je ferai donc référence à cette édition en 3 volumes (Paris, Cuchet, 1789).

Cet ensemble d'écrits, parfaitement délimité et cohérent, est donc le résultat d'un travail collectif, non d'une équipe de chercheurs unissant leurs efforts pour éclairer tel ou tel phénomène ou ordre de phénomènes, mais de quatre esprits qui se reconnaissent liés par la conviction où ils sont parvenus à un moment crucial de l'histoire de leur discipline, qu'on ne peut avec une langue qui retarde, avoir une connaissance qui avance. Le conflit entre l'archaïsme de l'une et la nouveauté de l'autre leur est de plus en plus sensible et insupportable. Réformer la langue, donc, mais comment l'édification d'une nouvelle nomenclature ne poserait-elle pas le problème de la relation d'interdépendance entre la connaissance et son support linguistique? Ce problème d'ordre épistémologique était au centre des réflexions de Lavoisier et de ses amis, tout au long des très nombreuses séances de travail qu'ils ont tenues de 1782 à 1787. Mais les chimistes intéressés ont très tôt compris que, pour venir à bout de ces difficultés d'ordre supérieur, il ne suffisait pas d'unir leurs compétences: il fallait avoir recours à une recherche pluridisciplinaire, en s'associant les représentants d'autres sciences plus avancées dans l'analyse de leurs rapports avec la langue.

Une telle association ne pouvait que sembler naturelle à des hommes qui n'étaient pas de stricts spécialistes. Depuis plusicurs années Lavoisier avait collaboré avec des géologues comme Rouelle et Guettard, avec le mathématicien et physicien Laplace (réalisation du calorimètre et expériences de calorimétrie), avec le mathématicien et ingénieur Meusnier (décomposition de l'eau). Guyton de Morveau avait été un collaborateur de Buffon, notamment pour l'Histoire des Minéraux. Fourcroy avait publié une Entomologia Parisiensis, mise à jour de l'Histoire des Insectes de Geoffroy. De leur côté, Monge et 
Laplace avaient participé à des recherches et expériences de chimie. Si bien que Lavoisier peut écrire (I, XXIX, Discours Préliminaire) : «Si quelquefois il a pu m'échapper d'adopter, sans les citer, les expériences ou les opinions de M. Bertholet, de M. de La Place, de M. Monge, et de ceux en général qui ont adopté les mêmes principes que moi, c'est que l'habitude de vivre ensemble, de nous communiquer nos idées, nos observations, notre manière de voir, a établi entre nous une sorte de communauté d'opinions dans laquelle il nous est souvent difficile à nous-mêmes de distinguer ce qui nous appartient plus particulièrement.»

\section{UNE «MÉTHODE DE NOMMER» D'INSPIRATION CONDILLACIENNE}

S'ils ont ainsi pris conscience des déficiences de leur langue et de l'absolue nécessité de rétablir son aptitude à clarifier les concepts et stimuler la découverte, ils ont aussi trouvé un appui et une justification dans la philosophie générale de Condillac, dont ils n'ont pas hésité à se réclamer. Lavoisier notamment, à deux reprises, dans son Mémoire de 1787 comme dans le Discours préliminaire de 1789, insiste sur la «justesse», la «clarté», l'«évidence» des principes posés par le philosophe «dans sa Logique et dans quelques autres de ses ouvrages». Dès la première page (I, V) du Discours, il cite et résume les thèses essentielles de la Logique: «Il y établit que nous ne pensons qu'avec le secours des mots; que les langues sont de véritables méthodes analytiques; que l'algèbre la plus simple, la plus exacte et la mieux adaptée à son objet de toutes les manières de s'énoncer, est à la fois une langue et une méthode analytique; enfin que l'art de raisonner se réduit à une langue bien faite. Et il en donne pour preuve son propre exemple: «...tandis que je n'avais pour objet que de perfectionner le langage de la chimie, mon ouvrage s'est transformé insensiblement, sans qu'il m'ait été possible de m'en défendre, en un Traité élémentaire de Chimie.»

La Logique est le dernier ouvrage publié par Condillac, l'année même de sa mort (1780). C'est une sorte de condensé de toute son œuvre, rédigé à la demande du comte Potocki à l'usage des étudiants lithuaniens. Rappelons qu'il avait déjà composé tout un Cours d'études pour l'infant de Parme dont il avait été le précepteur. Il savait donc, à l'occasion, donner à sa pensée un tour didactique, que Lavoisier apprécie en parlant de la Logique comme d'un «ouvrage que les jeunes gens qui se destinent aux sciences ne sauraient trop lire» (III, 8). Ami des Encyclopédistes, Condillac apparaissait comme ayant repris avec plus d'ampleur et de rigueur les thèses (dites plus tard sensualistes) de Locke, dont Voltaire avait fait l'éloge en commençant par cette phrase mémorable (Lettres Philosophiques, XIII): «Tant de raisonneurs ayant fait le roman de l'âme, un sage est venu qui en a fait modestement l'histoire.» C'est aussi l'«histoire de l'âme» que fait Condillac dans son Essai sur l'origine des connaissances humaines (1740), puis dans le Traité des sensations, pour ne parler que des ouvrages les plus marquants; toute la genèse de l'entendement humain se développe à partir $\mathrm{d}^{\prime}$ '«un seul principe», qui est la sensation.

De cette genèse, qui occupe en particulier la première partie de la Logique, il suffit de retenir ici le chapitre IV, «Comment la nature nous fait observer les objets sensibles pour nous donner des idées de différentes espèces», et une partie du résumé que l'auteur en donne: «Nos premières idées sont des idées individuelles. En classant les idées on forme des genres et des espèces. Les idées individuelles deviennent tout à coup générales. Les idées générales se soudivisent en différentes espèces... Toutes nos idées se font avec la même méthode, et cette méthode est l'analyse.» C'est surtout la seconde partie qui a pu orienter les réflexions et le travail des auteurs de 1787: «L'analyse considérée dans ses moyens et dans ses effets; ou l'art de raisonner réduit à une langue bien faite.» Une certaine théorie critique de la connaissance a été déterminante dans le domaine de la création lexicale, ouvrant la voie à une néologie méthodique et lourde de sens. 
Les meilleurs chimistes, tant français qu'étrangers, considérant la nomenclature traditionnelle, en étaient arrivés à dresser un constat d'inaptitude. Outre le vocabulaire ésotérique hérité des alchimistes, qui contribuait, dévoyant les recherches, à égarer la chimie dans l'occultisme, il était de plus en plus évident qu'on était en présence d'une nomenclature non scientifique, puisqu'une même réalité chimique (au regard des découvertes actuelles) était désigné par des noms entièrement différents, et qu'un même nom désignait des réalités qui (au regard des analyses récentes) étaient totalement différentes. D'où un blocage de tout échange entre savants, national et international. Les malentendus (dont les sciences dites humaines nous offrent tant de désolants exemples) doivent être bannis des sciences dites exactes. «C'est, dit Lavoisier, Bergman et Macquer qui ont sollicité cette réforme. Le savant professeur d'Upsal, M. Bergman, écrivait à M. de Morveau, dans les derniers temps de sa vie, ne faites grâce à aucune dénomination impropre.» (I, 26). Et les réformateurs de 1787 ne pouvaient que souscrire au verdict sévère de Condillac: «Faut-il s'étonner qu'on ne sache pas raisonner, quand la langue des sciences n'est qu'un jargon composé de beaucoup trop de mots, dont les uns sont des mots vulgaires qui n'ont pas de sens déterminé, et les autres des mots étrangers ou barbares qu'on entend mal?» (Log., II, 7, p. 166). L'auteur de l'Avertissement sur les deux synonymies (III, 110) déplore l'imperfection du vocabulaire de certaines sciences, «en particulier la botanique et la minéralogie dans lesquelles l'immense quantité de noms différents donnés à une même chose a produit une confusion et une obscurité, que les travaux des hommes les plus infatigables n'ont point encore pu éclaircir». Il faut que la langue de la chimie, langue «mal faite», n'entrave plus la marche de cette science en plein renouveau. Les réformateurs en sont convaincus, mais l'opinion n'est pas unanime, on doit le reconnaitre: dans le Rapport sur la nouvelle nomenclature, les quatre académiciens qui en ont été chargés n'hésitent pas à écrire que cette science «s'exprime depuis un demi-siècle avec une merveilleuse clarté»...

Quoi qu'il en soit, comment construire une langue «bien faite»? Les auteurs de la nouvelle nomenclature ne manquent pas de souligner qu'elle est «méthodique». C'est ce que Lavoisier a exprimé mieux que personne dans un passage capital de son Mémoire du 18 avril: «cette nomenclature nouvelle, pourvu qu'elle ait été entreprise sur de bons principes; pourvu que ce soit une méthode de nommer plutôt qu'une nomenclature, elle s'adaptera naturellement aux travaux qui seront faits dans la suite; elle marquera d'avance la place et le nom des nouvelles substances qui pourront être découvertes et elle n'exigera que quelques réformes locales et particulières» (III, 17 et I, 186). Méthode de nommer, cette expression est reprise par Morveau (III, 39), par Foucroy (III, 81), et c'est bien là l'innovation géniale des quatre réformateurs, mise au point, on l'a vu, avec le concours des meilleurs esprits de l'époque, «après avoir profondément médité sur la métaphysique des langues» (III, 5). Le Traité de chimie de 1789 sera une illustration lumineuse de cette méthode, sur laquelle Lavoisier revient souvent, pour en faire percevoir l'adéquation non seulement aux connaissances actuelles, mais aussi aux découvertes futures. S'il ne s'agissait que de refléter un certain état de la science, la réforme se réduirait à un tableau statique, alors qu'il s'agit évidemment, dans l'esprit des auteurs, d'une démarche intellectuelle apte à préserver et accompagner l'avenir de la science.

La méthode de nommer, comme les dénominations qui en procèdent, sont inséparables d'une classification des objets à nommer, en l'occurrence les corps chímiques. Les analyses auxquelles aboutissaient les diverses opérations et expériences des nomenclateurs de 1787 et des chimistes dont ils reconnaissaient les travaux permettaient de classer ces corps dans un Tableau, complet et détaillé, présenté à l'Académie des Sciences. N'est-ce pas Condillac qui affirmait ( $\log$., II, 5) : «Nous n'avons de nouvelles idées que parce que nous formons de nouvelles classes.» Mais il précisait: «Convaincus que les classes ne 
sont que des dénominations, nous n'imaginerons pas de supposer qu'il existe dans la nature des genres et des espèces, et nous ne verrons dans ces mots, genres et espèces, qu'une manière de classer les choses suivant les rapports qu'elles ont à nous et entre elles.» Position aussi prudente que modeste, qui sera aussi celle des nomenclateurs quand, par exemple, réunissant dans une première grande classe les substances «simples», ils soulignent qu'elles «ne sont simples pour nous que parce qu'on n'a pas encore pu en faire l'analyse» (III, 82): c'est en quoi elles ont un rapport «à nous et entre elles», selon la remarquable formule de Condillac. De même, dans son Traité, Lavoisier dit des «terres» qu'on peut les regarder comme des êtres simples «en attendant de nouvelles découvertes $(I, 172)$, mais il soupçonne que «ces substances pourraient bien n'être que des métaux oxidés» (I, 180), ce qui sera avéré une vingtaine d'années plus tard. Dans le Tableau de 1787 , les «substances non décomposées» se présentent sur deux colonnes, la seconde comprenant ces substances «mises à l'état de gaz par le calorique». Il y a, en opposition avec cette première grande classe de «non décomposées», une seconde grande classe, celle de ces substances «combinées», subdivisée en «combinées avec l'oxygène», «oxygénées gazeuses», "oxygénées avec bases» et «combinées sans être portées à l'état d'acide». Il faut, maintenant, examiner les solutions onomasiologiques apportées par les auteurs dans le cadre de cette classification : autrement dit, les bases proprement linguistiques de leur nomenclature.

Auparavant, je voudrais ouvrir ici une parenthèse. Dans Les mots et les choses (1966), Michel Foucault soutient qu'à partir du XIX ${ }^{\mathrm{e}}$ siècle, «l'étude de l'organisme prend le pas sur la recherche des caractères taxinomiques» (p. 14), et dans le brillant chapitre 5 , intitulé «Classer», de sa première partie, tout en citant Condillac à plusieurs reprises, il ne fait pas la moindre mention de la nomenclature chimique de 1787. Certes, l'objet de sa recherche, ce sont les vivants et la vie, mais comment oublier que l'extraordinaire nouveauté de la chimie de Lavoisier est précisément son entrée dans la physiologie (combustion, respiration, sang) et que les substances végétales et animales sont le «noyau dur» de sa recherche, en même temps qu'elles posent à sa nomenclature les plus épineux problèmes? En ce domaine les résistances qu'il rencontre sont grandes: en 1803, dans son Histoire naturelle des végétaux, écrite en collaboration avec Mirbel, l'illustre Lamarck, auteur des deux volumes de l'Introduction (Botanique générale et Histoire de la botanique), dénonce les "principes de la chimie moderne qui ne voit partout qu'oxygène et opération d'oxygène» (t. I, p. 222) et se plaint (p. 216) de «l'influence de la chimie moderne» et de ses «hypothèses contradictoires». Une telle incompréhension a de quoi surprendre, mais Lamarck a au moins le mérite d'avoir bien vu que la chimie nouvelle, qu'il refusa, liait l'oxygène aux phénonèmes de la vie. On ne peut souscrire à cette affirmation de Foucault : «Jusqu'à la fin du XVIII' siècle, la vie n'existe pas, mais seulement des êtres vivants.» (p. 173)

\section{LE PRINCIPE DE LA DÉNOMINATION MOTIVÉE}

Dans le cas des substances dites (provisoirement) simples, au nombre de 55, plusieurs solutions ont été retenues: l'une, conservatrice, dans le cas des métaux, dont les noms sont «plus employés dans les arts et dans la vie civile» (au nombre de 17); on ne touche pas aux noms consacrés par le travail et les échanges, l'or, le fer, le zinc, etc., parce que, outre qu'ils sont recommandés par l'ancienneté de leur usage, ils sont des unités lexicales simples, et non des syntagmes périphrastiques. L'autre solution, celle-là néologique, s'inspire du même principe : «'ai désigné autant que je l'ai pu les substances simples par des mots simples», dit Lavoisier (I, XIX). Et plus loin (I, 53): «Nous nous sommes attachés surtout à n'admettre que des mots courts et, autant qu'il était possible, qui fussent susceptibles de former des adjectifs et des verbes.» Toute désignation périphrastique est «embarrassante dans le discours» (I, 32) et s'oppose à la formation des dérivés et composés que le nom 
des substances simples suscitera nécessairement. On dira donc calorique et non matière de la chaleur; oxygène et non air vital; hydrogène et non gaz inflammable; azote et non air phlogistiqué ou vicié; silice et non terre siliceuse; alumine et non terre base de l'alun; baryte et non terre pesante; chaux et non chaux vive; carbone et non charbon pur. Ceci pour des raisons de commodité morphologique, en dehors des raisons d'ordre théorique, plus impérieuses, qui seront bien entendu invoquées. En effet, des noms comme silice, alumine ou carbone sont seulement extraits de la base latine de mots déjà existants ce qui répond d'ailleurs très bien à leur caractère de "principe». Mais il n'en est pas de même de noms comme oxygène, hydrogène ou azote.

Car dans ce cas, comme dans celui des substances décomposables, les nomenclateurs veulent éviter de former «ces mots tout-à-fait insignifians qui ne se relient à aucune idée connue» (III, 36). Ces mots «insignifiants» et arbitraires seraient déplacés dans une néologie raisonnée et méthodique. Les créations lexicales laisseront apparaître leur motivation interne: «Nous avons fait en sorte d'exprimer par ces nouveaux noms la propriété la plus générale, la plus caractéristique du corps qu'ils désignaient» (III, 19): celle de l'oxygène est de «former des acides», celle de l'azote est de «priver de vie les animaux qui respirent ce gaz» $(\mathrm{I}, 55-6)$, celle de l'hydrogène d'être "générateur de l'eau». C'est une des propriétés qui est choisie, comme indiscutable, de façon à assurer une validité durable à la dénomination. Reconnaissons qu'ils ne se sont pas trompés, et que ces noms, et celui du carbone, sont ceux des principaux constituants de la matière vivante, depuis plus de deux siècles. Notons en passant que le principe d'exclure les mots dits «insignifiants» n'a pas été toujours admis par les chimistes. Ainsi Thénard, dans son Traité de Chimie (4 éd., 1824, t. I, p. 166) critique le mot oxygène, devenu impropre puisque tout acide n'est pas oxacide, et souligne «l'avantage qu'il y aurait à donner des noms insignifians aux corps simples». Une dénomination arbitraire est en effet tentante, de par sa neutralité même. C'est ainsi qu'on a vu les physiciens introduire dans la nomenclature des particules élémentaires (proton, neutron, etc.) le mot mystérieux de quark, extrait de Finnegans Wake de Joyce! Ce mot aura-t-il autant de succès que le mot gaz, lui aussi inventé, il y a trois siècles, par Van Helmont? Les termes nouveaux dont les sciences ont évidemment besoin au cours de leur développement ont-ils plus de chances de durer si la dénomination est arbitraire ou si elle est motivée? Ce problème onomasiologique mériterait d'être débattu, car le choix de la solution retenue n'est pas indifférent. Notons que la siglaison et l'acronymie peuvent, pour ainsi dire, neutraliser cette opposition, en aboutissant à des signes réellement motivés mais apparemment arbitraires (laser, sida, etc.). En tout cas, Lavoisier et ses amis ont pris clairement position.

Certes, des noms ont été discutés, en particulier ceux qui étaient construits sur des bases grecques. Dans leur Rapport sur la nouvelle nomenclature, les Académiciens dénoncent des étymologies «prises souvent dans une langue ancienne, déjà presqu'ignorée des savans, et dans laquelle il ne peut y avoir ni trace, ni notion quelconque des choses, ni des idées qu'on doit leur faire signifier». C'était là poser un problème qui mérite qu'on s'y arrête; incontestablement, la «méthode de nommer» des réformateurs comporte ce recours au grec, recours qu'ils ne manquent aucune occasion de signaler, mais qu'ils n'expliquent pas.

S'agissait-il d'une langue «déjà presqu'ignorée des savans»? Depuis la Renaissance, le grec avait longtemps joui d'un immense prestige. Rabelais fait dire à Gargantua, dans sa lettre à son fils Pantagruel, que cette langue «sans laquelle c'est honte que une personne se die savant», est la première qu'il doit apprendre. Peut-être cet engouement pour le grec était-il moins vif, mais dire que le grec était presque ignoré des savants est absurde. Cette langue ne pouvait être étrangère aux mathématiciens et aux physiciens (Monge et Laplace ont participé aux «conférences» des nomenclateurs), ni aux médecins 
et anatomistes, qui devaient aux auteurs grecs la majeure partie de leur vocabulaire, ni, pour la même raison, aux grammairiens et rhétoriciens, ni aux naturalistes, en particulier l'illustre Linné qui, en Botanique surtout, avec son Système Sexuel, avait proposé une classification des plantes en 24 classes qui toutes avaient reçu des dénominations d'origine grecque, habillées en latin scientifique: monandria, diandria, triandria... polyandria, didynamia, tetradynamia, monadelphia... polyadelphia, syngenesia, gynandria, monoecia, dioecia, polygamia, cryptogamia, sans parler des subdivisions monogynia, digynia... gymnospermia, angiospermia, etc. Le plus remarquable, dans ces appellations, c'est que si quelques-unes étaient empruntées (forme et sens) ou adaptées (forme seulement) du grec, la plupart étaient de véritables productions, associant de manière inédite des morphèmes d'origine grecque auxquels effectivement, comme le disent les auteurs du Rapport, on «fait signifier» en liaison ce qu'ils ne signifiaient pas en liberté. D'ailleurs, sans qu'il soit besoin de puiser dans une source étrangère, toute langue est amenée à aller chercher dans son fonds propre de très nombreux mots qui dans l'état de langue auquel ils appartenaient, avaient un contenu tout autre que celui dont, souvent quelques siècles plus tard, ils se trouvent chargés.

La critique des Rapporteurs est donc peu fondée, puisque le grec a déjà pris une place importante dans le vocabulaire scientifique de l'époque. Mais s'ils réagissent, c'est que celui de la chimie est encore intact et qu'ils redoutent la contagion. Ils livrent, sans grande conviction, un combat d'arrière-garde, et disent «attendre l'épreuve du temps», mais le temps ne pouvait que leur donner tort. C'est en vain que les opposants vont chercher chicane aux réformateurs en prétendant, paradoxalement, être meilleurs hellénistes. Lavoisier s'en amuse (I, 94): «On a critiqué même avec assez d'amertume cette expression hydrogène, parce qu'on a prétendu qu'elle signifiait fils de l'eau et non pas qui engendre l'eau. Mais qu'importe, si l'expression est également juste dans les deux sens ?» Il est tout à fait exact que Lavoisier et ses amis ont donné à ce morphème -gène

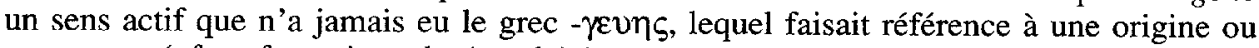
une nature ( $c f$. en français endogène, hétérogène). En «faisant signifier» pour la première fois autre chose, à savoir «qui engendre», à cet élément -gène (dans oxygène et hydrogène), les réformateurs ouvraient un système de dénomination extraordinairement productif, et dans les domaines les plus divers, comme on peut le constater aujourd'hui.

Faut-il donc s'étonner que les réformateurs de 1787 n'aient pas jugé bon de justifier ce recours au grec ? En participant à ce travail, collectif et pluridisciplinaire, d'où est sortie la nouvelle nomenclature, les divers savants, attachés à «l'uniformité de langage si essentielle à la communication des travaux et aux progrès de la science» (III, 28), ne pouvaient manquer de s'accorder sur la nécessité, pour la chimie, de contribuer à son tour à l'internationalisation du langage scientifique, déjà bien avancée. Les chimistes n'auraient pas à défendre jalousement leurs langues respectives si se constituait et se répandait un vocabulaire, commun aux diverses sciences comme aux diverses nations, exploitant les ressources d'une langue ancienne dont la redécouverte en Occident avait tant contribué à l'essor des connaissances et la diffusion des «lumières». Cette langue, outre l'avantage d'être souvent connue dès le temps des études, séduisait par son aptitude à réduire toute une «phrase» (on appelait ainsi une séquence codée de plusieurs mots) à une seule unité lexicale: les éléments de formation et leurs modèles de combinaison sont en grec d'une remarquable variété. Le très influent Rollin, professeur d'éloquence au Collège Royal, dans sa Manière d' enseigner et d'étudier les Belles-Lettres (1740), avait célébré «l'admirable fécondité de cette langue, qui par la dérivation et la composition des mots se multiplie presque à l'infini» (I, 2). De même, le naturaliste Duméril, dans sa Zoologie Analytique (1806), dira, pour justifier ses classifications néologiques: «c'est principalement la dénomination tirée du grec que nous avons adoptée.» (p. XXI) Ces modèles grecs, une 
fois acclimatés, avec les variantes phonétiques imposées, dans telle ou telle langue moderne, se prêtent en effet à toutes sortes de constructions nouvelles.

\section{LE RECOURS À LA SUFFIXATION}

Venons en maintenant aux substances «combinées», c'est-à-dire celles qui (en dehors de l'azote, du carbone, du soufre et du phosphore «que l'on a pu obtenir simples») ne sont «connues que dans leurs combinaisons avec l'oxygène». Mais «nous les avons séparés de l'oxygène par la pensée, et nous les supposons dans leur état de pureté auquel il est vraisemblable que l'art parvienne à les réduire». Il y a donc, par hypothèse, à la base de tout acide, un «radical»: ainsi l'acide acétique, oxalique, etc. est considéré comme une combinaison d'un radical acétique, oxalique, etc. avec l'oxygène. Le mot radical, équivalent de base acidifiable (III, 40), employé d'ailleurs depuis une vingtaine d'années par les linguistes, était introduit dans le vocabulaire chimique par Guyton de Morveau (I, 69); on sait qu'il se maintiendra, même quand le concept qu'il exprime sera élargi et enrichi. Ces radicaux, combinés avec l'oxygène, forment, comme les substances simples, des combinaisons binaires (I, 207), acides ou oxides, à la dénomination desquelles les nomenclateurs de 1787 vont apporter une solution à la fois moderne et originale, assez originale d'ailleurs pour traverser toutes les étapes de la chimie, qu'elle y apparaisse ouvertement ou en filigrane. Le principe général étant d'exclure les mots «insignifiants», comme on a vu, de «n'admettre aucun mot sans y attacher une idée» (III, 19), il faudra, pour les combinaisons, que leur nom laisse apparaître les éléments qui les constituent: «nous les avons désignés par des noms composés comme le sont les substances elle-mêmes» (I, XX). La méthode de nommer repose donc sur une sorte de parallélisme chimico-morphologique. Elle est aisément applicable dans le cas des combinaisons binaires: ainsi les acides entreront dans une nomenclature binaire à deux termes, dont le premier «est le terme générique d'acide», et «le second spécifie chaque acide» (III, 90), ce terme spécifique, tiré des noms des substances dites simples ou radicaux, permettant de edifférencier les acides les uns des autres». Ce second terme sera ce que nous appellerions aujourd'hui un adjectif de relation, dérivé d'un nom déjà ancien en français (gallique) ou savant, latin ou grec (malique, oxalique). Comment ne pas reconnaître ici les principes classificateurs de Condillac, et plus encore, l'exemple du grand classificateur du Systema naturae, dont 12 éditions successives, de son vivant, avaient affirmé l'autorité ?

Mais c'est dans ce cadre, déjà dessiné, proposé et accepté par certaines sciences, que va intervenir une innovation lexicale aux conséquences immenses, que les progrès de la chimie pendant deux siècles ne feront que consacrer avec éclat. Cette innovation, c'est l'introduction dans la nomenclature, d'une suffixation qui n'est pas seulement de nature grammaticale, mais plus encore de nature sémantique, permettant la classification fine et l'identification des réalités chimiques à désigner.

Il est clair que c'est en travaillant à la dénomination des acides que Lavoisier et ses amis ont eu l'idée de cette néologie révolutionnaire. L'analyse chimique les mettait en présence de deux «proportions différentes» de «principe acidifiant» et de «principe acidifié», lesquelles «constituent toutes des points d'équilibre ou de saturation» (I, XXII): il peut y avoir une forte, ou une faible proportion d'oxygène, d'où une différence de comportement des acides. Considérant «les noms déjà employés de vitriolique et de sulfureux» avec le générique acide (III, 92), les réformateurs vont utiliser cette différence de «terminaison» en -ique et en -eux pour former deux types de termes spécifiques, le premier (sulfurique, acétique, phosphorique...) dans le cas où la base acidifiable est complètement saturée par l'oxygène, le second (sulfureux, acéteux, phosphoreux) dans le cas inverse. Telle est la règle «aussi générale que simple» qui sera suivie dans la dénomination des acides. Une 
simple variante de terminaison adjectivale acquiert le statut de fait linguistique pertinent, donnant naissance à deux morphèmes distinctifs.

Cette innovation dans la «méthode de nommer» va s'étendre, par une sorte de réaction en chaîne, à la désignation d'autres combinaisons: d'abord celle des «substances oxygénées avec bases» ou «sels neutres» en général (III, 97). Les noms de ces composés salins seront terminés en -ate lorsqu'ils contiennent un acide saturé (nom en -ique), en -ite quand l'acide n'est pas saturé d'oxygène (nom en -eux); ainsi «leur nature n'est indiquée que par deux mots» (III, 97). Cela ne signifie pas qu'on connaisse dans tous les cas ces deux types de sels; si sont signalés des sulfates et des sulfites, des phosphates et des phosphites, etc., à certains acides ne correspondent que des sels en -ate (carbonates, citrates, etc.), à d'autres que des sels en -ite (tartrites, nitrites, etc.). Cette «uniformité de terminaison», tout en visant à «exprimer des composés analogues» rangés clairement dans la même classe, est évidemment apte à exprimer des composés non encore connus qui viendront occuper les cases restées vides; Fourcroy calcule (III, 100) qu'on peut estimer à 722 le nombre de sels neutres «dont les dénominations peuvent être formées méthodiquement».

Seconde application de la dénomination de type suffixal : la dernière colonne du Tableau est réservée aux «substances combinées dans leur état naturel, et sans être oxygénées ou acidifiées». En dehors des alliages et amalgames, dont les noms sont conservés, on trouve les carbures, les sulfures et les phosphures, dont les noms «ne diffèrent que par la terminaison de noms très connus», cette même terminaison faisant comprendre qu'il s'agit de «composés analogues». Beaucoup d'autres noms en -ure viendront plus tard s'ajouter à la liste de 1787, la caractéristique de cette méthode étant, il faut le répéter, de rester ouverte comme un «modèle».

Avant de poursuivre l'analyse de la méthode onomasiologique des nomenclateurs de 1787 , il convient de s'arrêter un peu sur ce procédé suffixal de classification et de dénomination, dont la fécondité en chimie a été extraordinaire.

En effet, en dehors de l'exploitation, qui s'est poursuivie, des suffixes -ique et -eux des acides, -ate et -ite des sels, -ure des combinaisons non oxygénées (un peu plus tard considérées comme sels d'hydracides), les chimistes ont fait appel à de nouveaux suffixes à vocation d'identificateurs. Le premier, employé de façon tout à fait anarchique et abusive, remplissait mal la fonction, dévolue à ces morphèmes par les réformateurs de 1787 , de signe de reconnaissance de la nature chimique du corps ainsi désigné : il s'agit du suffixe -ine qui sert depuis près de deux siècles à désigner des «substances» extrêmement diverses, associé à une base qui désigne l'origine (quinine), la propriété (dextrine), l'effet (narcotine) de la substance en question; encore faut-il signaler que le nom même de l'inventeur peut servir de base (Fuchs, trad. de Renard, pour fuchsine), ce qui nous ramène aux désignations de type éponymique de l'ancienne nomenclature (sel de Sylvius, de Glauber...), type qui, soit dit en passant, est abondamment représenté dans le vocabulaire médical d'aujourd'hui avec les maladies, syndromes, etc. de $X$, ou $Y$, ou $Z$ ! Cette sorte d'adaptabilité et, pour ainsi dire de désinvolture du suffixe -ine (largement exploitée dans les appellations commerciales des produits pharmaceutiques), n'a pas tardé à inquiéter les chimistes. Naturellement, on pouvait le faire remonter aux auteurs de 1787, qui avaient appelé alumine la terre base de l'alun: toute «substance de base», tout «principe immédiat» semblait se prêter à cette suffixation. Ainsi pouvait-on voir apparaître, dans les années qui suivirent, l'albumine (1792), la fibrine (1794), l'asparagine (1807), l'inuline (1807), la stéarine (1816), la strychnine (1818), etc. Devenu «insignifiant», aux yeux de chimistes scrupuleux, il va être rayé de certaines appellations. En 1832, Wöhler et Liebig, dans leur Recherche sur le radical de l'acide benzoique (Ann. de Ch. et Phys., LI, p. 273), proposent d'exprimer ce «radical composé» par le suffixe (et infixe) $-y l(e)$, dans benzoyle. Aussitôt Berzelius, 
dans une lettre datée du 2 septembre, les félicite (loc. cit., LI, p. 308) et les auteurs précisent dans une note: «Nous avions d'abord choisi le nom de benzoïne ... nous l'avons changé contre benzoyle... La terminaison -yle est bonne en ce qu'elle s'éloigne des terminaisons en -ine, telles que dans strychnine, salicine, etc.» Pareillement, en 1833, dans son Mémoire sur les camphres artificiels (Ann. de Ch. et Phys., LII, p. 400), Dumas en dénonce l'ambiguïté: «On sent assez combien il est fâcheux que la morphine, l'inuline, la caféine, la naphtaline, l'allantö̈ne, etc., substances qui n'ont entre elles pas plus d'analogie que le chlore et le sulfure de plomb, soient pourtant désignées par des noms qui offrent à l'esprit l'idée d'une réunion dans la même famille.» En conséquence, après avoir en 1831 proposé camphogène, il ne retient pour les «hydrogènes carbonés», que «des noms terminés en ène, pour éviter la confusion avec les alcalis végétaux en ine comme quinine», et il précise : «chose nécessaire, car, pendant longtemps tout au moins, tout l'artifice de la nomenclature organique reposera sur des modifications dans les désinences.» D'où les noms de camphène et de citrène, suivis en 1834 par méthylène, pour en arriver à la nomenclature systématique de Laurent (1836), qui remplace benzine par benzène, naphtaline par naphtalène, etc. (Ann. de Ch. et Phys., LXI, p. 125). Il arrivera à cette suffixation en -ine, jugée trop laxiste, un peu ce qui est arrivé à certains genres linnéens d'une extension excessive, que les naturalistes ont dû «démembrer» en plusieurs genres d'une homogénéité plus satisfaisante. Il semble alors qu'on puisse recourir à cette «désinence» comme à un procédé commode mais provisoire de désignation, dans l'attente d'une analyse plus fine du corps considéré : c'est ainsi que la cholestérine de Chevreul (1816) deviendra le trop fameux cholestérol, rangé par son suffixe dans le groupe des alcools.

En effet, les progrès de la chimie (notamment organique) vont introduire dans la nomenclature, conformément à la «méthode de nommer» de 1787, des suffixes nouveaux qui sont autant de signes de reconnaissance, et des moyens d'échapper à la suffixation fourre-tout en -ine. Mais il ne s'agit plus comme en 1787, d'une création, à l'origine, délibérée et destinée à la constitution d'unités lexicales désignant des «substances congénères» (pour reprendre l'expression de G. de Morveau, III, 57). Il s'agit, le plus souvent, d'une dénomination (répondant à une découverte ou une exigence ponctuelle), dont la séquence phonétique terminale suggérera une utilisation systématique en tant que morphème. Par exemple, dans un Mémoire intitulé Recherches sur la nature et les propriétés chimiques des sucres (Comptes rendus de l'Ac. des Sc. 1838, VII, p. 109), Péligot propose d'appeler glucose «le sucre de raisin, celui d'amidon, celui de diabètes et celui de miel» qui constituent un seul et même corps. Il précise dans une note que le mot est

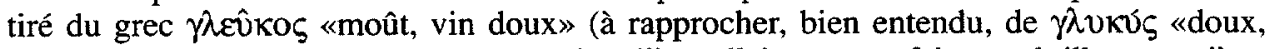
sucré»). Cette terminaison en -ose, assez singulière, allait pourtant faire une brillante carrière. Dès 1839 elle passait au rang de suffixe dans la désignation d'un corps considéré par Payen comme isomère avec l'amidon, la cellulose; en 1847, dans maltose; en 1860, dans la classification systématique des sucres par Berthelot (lévulose, saccharose, etc.); et, par la suite, dans de très nombreuses dénominations. Enfin ce morphème lié devient unité lexicale: les sucres ainsi nommés constituent le groupe des oses (nom attesté dès 1913 dans le manuel de Chimie organique de Moureu, $4^{e}$ éd.). Cette lexicalisation est si évidente que ose pourra un peu plus tard devenir base d'un dérivé, oside (avec les composés holoside, hétéroside et osidase).

Nous avons là un modèle d'évolution linguistique dont l'étape ultime, à savoir la constitution du morphème lié en unité autonome, est relativement rare; mais la transformation d'une simple séquence terminale en morphème est au contraire des plus fréquentes dans la nomenclature chimique: j'en ai donné de nombreux exemples dans mon Dictionnaire des structures du vocabulaire savant, auquel on voudra bien se reporter. Observons seulement ces séries de termes «congénères», où la communauté de suffixe traduit une 
parenté de contenu, ces séries de suffixés en -ine, -éne, -ane, -one, -yne, -ide, -ile, -ime, -ite, -yle, -ol, -ose, -ure, -al, -ate, etc.: elles autorisent, et à la limite imposent, une espèce de vue stratigraphique du vocabulaire de la chimie; une succession de couches, identifiables par leur marqueur suffixal, sont les témoins des différents âges de cette science, de l'éocène de 1787 à nos jours, et au delà. Comme en géologie, tout n'est pas d'une parfaite régularité : on est surpris par des affleurements, des disparitions, des réapparitions, des métamorphismes. Un renouvellement, parfois même un renversement de certaines conceptions chimiques, est sensible dans l'emploi des suffixes; j'ai esquissé, par exemple, l'histoire du suffixe -ol, qui mériterait une étude diachronique beaucoup plus précise. (D'une manière générale, la lexicologie des sciences offre aux chercheurs une mine de sujets de réflexion, inexplicablement négligés.) En recourant à la suffixation, Lavoisier et ses amis étaient conscients qu'ils avaient ouvert une voie royale à la nomenclature. À la dernière page de son Mémoire de 1787 (III, 108), Fourcroy n'hésite pas à écrire: «On peut donc assurer que ce n'est presqu'entièrement que par des terminaisons nouvelles que notre nomenclature diffère de l'ancienne.» Retenons le «presqu'entièrement», car on va voir qu'il y a une innovation, moins facilement discernable, mais tout aussi déterminante.

Mais, pour en finir avec la pratique de la suffixation, il faut noter qu'elle va être un moment contrariée, et finalement confirmée, par une nouvelle couche d'unités lexicales en relation avec une théorie chimique révolutionnaire qu'on va très tôt (1816) appeler «atomistique». Lavoisier, génie lucide, déclarait à propos de la «fermentation acéteuse», que, si les faits principaux sont connus, «l'exactitude numérique manque» $(I, 161)$. C'est à ce manque que va tenter de remédier l'anglais Dalton, dès le début du XIX ${ }^{\mathrm{e}}, \mathrm{s}^{\prime}$ inspirant peut-être de certaines hypothèses de Higgins (1789). Dès 1807, les idées développées par Dalton dans ses cours furent exposées par Th. Thomson dans son System of chemistry; cet ouvrage fut traduit en 1809 (Paris, Bernard, 9 vol.) par Riffaut, avec une importante Introduction de Berthollet, relative notamment à l'«hypothèse ingénieuse de Dalton, par laquelle il explique les proportions constantes que l'on observe dans les éléments de quelques combinaisons». Selon Dalton «les éléments des corps sont disposés à s'unir atome à atome, et si l'un des éléments est en excès, cet excès suit un rapport qui doit être exprimé par quelque multiple simple du nombre de ces atomes». Berthollet signale que, par exemple, l'acide oxalique s'unit à la potasse dans deux proportions différentes selon Thomson, et que «Wollaston vient de publier (1808) plusieurs observations analogues». Dès lors, comment exprimer ces «proportions», sinon par des morphèmes numéraux ? Et ces morphèmes pouvaient-ils être autrement qu'en position de préfixes? De fait Wollaston, dans une communication de 1810 «sur les sels subacides et suracides», dont le Bulletin de Pharmacie (t. II, 1810, p. 425) donne un extrait, parle d'un binoxalate et d'un quadroxalate de potasse.

$\mathrm{La}$ «théorie atomistique» de Dalton étant admise, il fallait convenir que la suffixation ne suffisait plus en tant que «méthode de nommer». Les chimistes français se rallièrent aux préfixes numéraux. Gay-Lussac écrit en 1820 (Ann. de Ch. et Phys., XIII, p. 212): «Par bisulfite nous entendons un sulfite qui contient deux fois plus d'acide sulfureux que le précédent, ou deux atomes.» Il faut dire que Berzelius, dès 1816 (Ann. de Ch. et Phys., II, p. 331), tout en reconnaissant que «plusieurs chimistes distingués n'ont pas adopté les spéculations atomistiques», commençait à élaborer sa célèbre notation symbolique qui tenait compte de la théorie «daltonienne», puisque la lettre symbole de l'élément était accompagnée d'un exposant numérique dans la formule du corps composé. Traduisant un Examen de quelques composés de Berzelius (Ann. de Ch. et Phys., 1820, XIV, p. 379), Riffaut admire cette «méthode nouvelle et expéditive pour représenter la composition des corps». Berzélius retenait l'initiale (ou les premières lettres) du nom latin de l'élément et, pareillement, empruntait au latin les préfixes numéraux (sesqui-, bi-, tri-, quadr-). L'autorité 
du maître les a maintenus un quart de siècle, jusqu'à ce que les chimistes français, fidèles aux choix de 1787 (comme à ceux de Linné), aient préféré l'emprunt au grec. $D i$ - succède à bi-, dans diamide (1848); tetr(a)- à quadra-, dans tetracarbure (1838); penta-, hexa-, hepta-, également dans la classification des carbures d'hydrogène de 1838 par Couerbe. Mais le fait linguistique capital est que la suffixation va jouer un rôle entièrement nouveau: à ces préfixes numéraux vont être associés directement, sans l'intermédiaire d'une base, les suffixes chimiques distinctifs. Il s'agit là d'une structure morphologique d'une évidente témérité, dont le succès a été pourtant éclatant. La première, à ma connaissance, des formations de ce type remonte à 1836, quand Laurent dans sa Théorie des combinaisons organiques (Ann. de Ch. et Phys., LXI, p. 125) inclut entre le cétène et l'éthérène (hydrogène bicarboné) un composé qu'il appelle tétrène, parce que, dit-il en note, «son équivalent est égal à 4 x 4». On trouvera un peu plus tard pentène (1864), pentane (1878), heptène (1864), heptane (1878), octane (1878), pentose et hexose (1891), diol, triol, pentol et hexol (1913). Selon la théorie chimique en vigueur, le préfixe numéral exprime le nombre d'atomes, ou volumes, ou équivalents, etc. présents dans le composé dont le suffixe exprime la nature. Peut-on d'ailleurs parler dans ce cas de préfixes et de suffixes? Ces mots impliquent la présence d'une base, alors que nous avons ici affaire à deux «morphèmes liés» qui se trouvent «interliés». Une telle méthode de nommer, évidemment inconcevable en 1787 dans le détail de ses effets, suivait cependant la ligne initialement tracée.

\section{SUBSTANCES SIMPLES ET MULTIPLES : LA FONCTION DE REPRÉSENTATION}

On a vu que dans le cas des combinaisons binaires, les réformateurs les désignaient «par des noms composés comme le sont les substances elles-mêmes». C'est ce que j'ai appelé le principe du parallélisme chimico-morphologique. Mais ils ne pouvaient se dissimuler que cette solution se heurtait, dans le cas des combinaisons multiples, à de sérieuses difficultés. Lavoisier en convient très honnêtement, à propos des acides animaux (I, p. 128): «Une nomenclature qui entreprendrait d'exprimer à la fois ces quatre bases serait méthodique sans doute... mais cette cumulation de substantifs et d'adjectifs grecs et latins... semblerait présenter un langage barbare, également difficile à retenir et à prononcer.» Ces derniers mots soulignent, une fois de plus, l'avantage des dénominations aisément mémorisables et euphoniques. En calquant le mot sur la composition du corps chimique, on introduit dans le lexique une galerie de monstres. C'est une difficulté que rencontrera en particulier la chimie organique au milieu du XIX ${ }^{\mathrm{e}}$ siècle. W. Hofmann, dans un important Mémoire Sur la constitution moléculaire des bases organiques (trad. in Ann. de Ch. et Phys., 1851, t. XXXII, p. 108), parle d'un composé qui «peut être appelé iodure de méthylotriéthylammonium», et il ajoute: «C'est peut-être ici la place de réclamer de l'indulgence pour les sesquipedalia verba que j'emploie dans le courant de cet examen, et dont les pires sont, je le crains, encore à venir...» L'auteur, on le voit, ne manque pas d'humour et rappelle opportunément l'expression célèbre de l'Art Poétique d'Horace. De la même façon, rendant compte d'un ouvrage de Graebe (Ann. de Ch. et Phys., 1868, t. XV, p. 433), Wurtz parle de l'acide dichlorohydroquinodisulfonique, en accompagnant le mot d'un point d'exclamation!

Cependant, Lavoisier et ses amis refusent les «dénominations arbitraires à la manière des anciens» (I, 183). Quand on analyse les substances végétales et surtout animales, on est amené à concevoir des «radicaux composés», des «radicaux à quatre bases», hydrogène, carbone, azote et phosphore $(\mathrm{I}, 208)$. Deux bases sont constantes, hydrogène et carbone. On parlera donc de radicaux carbone-hydreux ou hydro-carboneux, la place dans le mot marquant le principe dominant. Le radical formique, qui en combinaison avec l'oxygène donne l'acide formique, ne porte que le nom de formique, mais implicitement est «composé» des deux bases constantes «auxquelles se joint presque toujours l'azote et souvent le 
phosphore» (I, p. 208). Ce nom de formique lui reste, encore qu'il ne s'agisse pas d'un radical simple. Car la présence de ces éléments n'étant alors que probable, et leurs proportions indéterminées, comment songer en 1789 à une dénomination, en principe souhaitable, fondée sur le parallélisme? Il suffit, provisoirement, d'une appellation qui ne soit pas «insignifiante», puisque cet acide dit formique a été effectivement extrait des sécrétions des fourmis à l'origine, et encore qu'il soit vraisemblable que plus tard on le découvre ailleurs. Un des soucis majeurs des nouveaux nomenclateurs est de ne pas anticiper sur le futur et de s'en tenir à ce qui, dans l'état actuel de leurs connaissances, est assez solidement établi pour n'être pas totalement remis en cause dans l'avenir. Une langue «bien faite», selon l'expression de Condillac, ne peut être une langue qui fasse obstacle à ces «progrès de l'esprit humain» dont Condorcet, avant de mourir, va dresser le tableau. La dynamique optimiste du siècle des lumières circule, patente ou discrète, à travers les écrits de ces quatre chimistes révolutionnaires. Mais elle s'accompagne d'une prudence et d'une sorte de modestie scientifique, inséparable de la conviction, où ils sont tous, d'une adéquation nécessaire entre la connaissance et la langue, l'une ne devant jamais précéder l'autre, et inversement.

Mais si, pour les combinaisons multiples, des dénominations provisoires sont préférables, qui ne préjugent pas des réponses futures aux problèmes, il n'en est pas moins vrai qu'on peut imaginer un type de réponses linguistiques à ces problèmes, et c'est en apportant, dans la chimie, ce type de réponses, que Lavoisier et ses amis ont créé un vocabulaire scientifique général qui, sans eux, aurait eu le plus grand mal à se constituer et à s'interpréter correctement. Car c'est à eux que nous devons un type d'opérations morphologiques sur lequel j'ai attiré l'attention dans l'Introduction de mon D.S.V.S. (pp. XII-XIII) et sur lequel je voudrais ici revenir, avec plus de précision, de détails et d'exemples. J'ai appelé «fonction de représentation» la fonction dévolue à certaines «formes réduites» d'unités lexicales de «représenter» des unités, à titre de «formants», dans des unités plus complexes, et $\mathrm{j}$ 'ai dit que cette innovation capitale remontait aux réformateurs de 1787 , en soulignant que cette espèce de réduction morphologique n'avait rien à voir avec les troncations familières du type vélo, prof ou bus.

Observons en effet de plus près la néologie relative aux substances simples ou binaires : assurément, elle admet certaines «libertés» auxquelles nous ne prenons plus garde tant elles semblent aujourd'hui naturelles, consacrées qu'elles sont par un usage de plus en plus extensif. On avait besoin de ces libertés, notamment pour former ces noms simples et courts pouvant servir de bases de dérivés et de composés. Il est remarquable que la première dénomination de l'ancien air vicié ou gâté ait été gaz azotique: c'est celle qui figure dans la Synonymie ancienne et nouvelle (III, p. 115 à 245). En appelant azotique ce gaz, les nomenclateurs proposaient un mot très régulièrement construit sur un modèle grec, de $\alpha$ privatif et $\zeta \omega \tau \imath \kappa \delta \zeta$ «vital». Mais dans les Mémoires présentés devant l'Académie des Sciences, le syntagme gaz azotique est ramené au nom minimal azote, par un artifice évident mais parfaitement justifié, puisque ce nom peut aisément entrer dans la construction de nouveaux mots et que, par ailleurs, la terminaison -ique est appelée par les auteurs à un rôle particulier, comme on a vu. Azote est donc «extrait» de azotique. Lavoisier avoue que ce nom «présente quelque chose d'extraordinaire» (I, p. 55) et que d'autres noms avaient été envisagés. Il est vrai qu'il ne s'agissait pas d'une simple dérivation régressive (comme ammoniaque de ammoniacal ou alumine de alumineux). On peut aussi noter que c'était prendre des libertés avec le grec que de tirer de $\beta \alpha \rho v \tau$ ऽ «pesanteur» le nom de la baryte, par une métonymie audacieuse; comme de donner au morphème -gène une valeur, on l'a dit, qui n'existait pas dans le grec.

Mais il y a plus que ces écarts d'avec un modèle ancien. Il faut en venir à des créations lexicales singulièrement hardies, dont le principe est la réduction d'éléments formels à 
des signes représentatifs. Un premier exemple est celui d'oxide. D'une façon légèrement embarrassée, G. de Morveau s'en explique (III, p. 59): «Nous avons formé le mot oxide, qui d'une part rappelle la substance avec laquelle le métal est uni, qui d'autre part annonce suffisamment que cette combinaison de l'oxygène ne doit pas être confondue avec la combinaison acide, quoiqu'elle s'en rapproche à plusieurs égards.» Lavoisier dira (I, p. 84): «Un premier degré d'oxygénation constitue les oxides.» Ce nom d'oxide (sur lequel de nombreux mots seront construits) ne semble pas avoir été discuté. Il était pourtant étonnant: ox- y «représentait »oxygène, et -ide se rapprochait de acide, mais sans le représenter à proprement parler. Oxide formait avec acide une paire d'une simplicité lexicale frappante, si peu régulière qu'en ait été la création, et l'un comme l'autre se prêtaient aisément à la dérivation.

Autre exemple de réduction, portant cette fois sur le radical du mot simple. Dans formique et formiate, la base latine formic- (formicare, formicatio, formicosus...), respectée dans l'ancien nom d'acide formicin (lat. formicinus), se trouve ramenée à form-, comme si dans le latin formica «fourmi» -ica était un simple suffixe. Pourquoi ? L'opposition -ique (acide) et -ate (sel) se trouverait fragilisée dans une paire formicique-formicate, le second terme semblant additionner les suffixes -ique et -ate. Car le modèle n'est pas citriquecitricate, ni oxalique-oxalicate, l'opposition étant réalisée au niveau citr-et oxal-. On ramènera donc, dans le cas des mots construits sur le latin formica, au niveau form-. Les chimistes, plus tard, ratifieront ce choix, avec formaldéhyde, formol, formyle, et avec, en position 2, l'élément -forme, utilisé en 1834 par Dumas (Ann. de Ch. et Phys., t. LVI, p. 113), pour désigner des combinaisons d'acide formique ou de formiate avec différents corps sous le nom de chloroforme, bromoforme et iodoforme (ce dernier mot étant encore aujourd'hui, dans les divers dictionnaires, interprété comme construit à l'aide de l'élément -forme de uniforme ou multiforme!).

Dans sulfate, sulfite et sulfure, la base latine sulfur- (sulfuratus, sulfureus...), de sulfur «soufre», respectée dans sulfureux et sulfurique, se trouve ramenée à sulf-, comme si -ur était un simple suffixe. Les nomenclateurs donnant au suffixe -ure un signifié particulier, il aurait fallu, sans réduction du radical, parler de sulfurure et de sulfurate (avec dérivés éventuels sulfururé et sulfuraté), tous mots que le «jugement de l'oreille» (III, 42 et 52) récusait (pour ne rien dire des calembours possibles).

Dans phosphate (proposé dès 1782 par Guyton de Morveau), phosphite et phosphure, la base phosphor- (du lat. scientifique phosphorus, adaptation du grec $\phi \omega \sigma \phi о \rho \sigma \varsigma ~ « q u i$ apporte la lumière»), d'où en français phosphore dès 1677 , respectée dans phosphorique et phosphoreux, est réduite à phosph-, ce qui constitue une atteinte particulièrement grave à la structure d'un mot composé, clairement motivé, dont le second morphème -phore, présent dès le $\mathrm{XVI}^{\mathrm{e}}$ siècle dans de nombreux mots d'origine savante, se trouve quasiment supprimé. Phosph- est donc chargé de représenter phosphor-, phosphorate et phosphorure étant écartés (probablement pour les raisons d'euphonie évoquées plus haut, les mots «simples et courts» étant d'ailleurs toujours préférés). Là encore, les chimistes ont ratifié les choix de 1787 .

De libertés en libertés prises avec la morphologie, on en arrive à la dernière étape, celle-ci décisive, où la fonction de représentation se manifeste pleinement. Dans son Mémoire de Mai 1787 (III, p. 53), Morveau rappelle que «les acides que l'on retire, par distillation, du tartre, des matières sucrées, du bois, etc. ont été nommés, par les chimistes, esprits empyreumatiques». Et il ajoute : «Il nous a paru important de faire entrer ce caractère dans leurs dénominations; mais pour en rendre l'expression d'un usage plus commode, nous l'avons réduite au dissyllabe pyro.» Ainsi l'esprit empyreumatique du bois devient l'acide pyroligneux, et ses sels des pyrolignites. Le mot dissyllabe n'est pas choisi au hasard: les cinq syllabes de empyreumatique sont réduites à deux, et le morphème pyro-, 
représentant empyreumatique, va fonctionner comme adjectif de ligneux, comme de tartareux et de muqueux. Ce morphème révolutionnaire va prendre une très grande importance dans la nomenclature chimique. C'est ainsi qu'il sera pris pour base par Laurent dans pyrène, par Anderson dans pyridine, etc. À cette occasion, il faut noter un fait remarquable : le remplacement, dans certains cas, de ce morphème préfixé pyro- par le morphème suffixé -one. En 1833, le chimiste Bussy (Ann. de Ch. et Phys., LIII, p. 399) avait obtenu à partir de l'acide margarique un corps qu'il avait appelé esprit pyromargarique; mais il abandonnait cette appellation, sur les conseils de Thénard et Chevreul, pour celle de margarone, et il ajoute: «D'après ce principe de nomenclature, les esprits pyro-acétique, stéarique, oléique devront être désignés par les expressions de acétone, stéarone, oléone.» Ainsi le nom de l'acétone (et des cétones) est né indirectement du «dissyllabe pyro»...

Cependant ce «représentant» pyro n'exprimait qu'une caractéristique du corps ainsi nommé, et non un corps associé à un autre dans un composé. Pour désigner des combinaisons multiples, d'un nom qui fasse apparaître les éléments constituants, il fallait qu'un ou plusieurs de ces éléments ne soient évoqués que sous la forme réduite de morphèmes représentant leurs noms respectifs. Dès lors, l'innovation était totale, et la voie ouverte à une nomenclature légère, dans laquelle la dénomination des corps composés se ferait autrement que par juxtaposition de noms intégralement exprimés.

Cette nouveauté n'apparaît pas dans les Mémoires dès 1787. C'est en 1789 que Lavoisier l'introduit, dans son Traité élémentaire de Chimie, en utilisant un morphème hydr(o)-, qui représente le mot complet hydrogène; le «Tableau des combinaisons binaires de l'Hydrogène avec les substances simples» (I, p. 216) présente une longue liste d'hydrures, combinaisons de l'hydrogène avec les métaux, les terres et les alcalis, ainsi que sa combinaison avec le carbone, sous le nom de radical hydro-carboneux ou carbone-hydreux. On a vu plus haut comment Lavoisier avait été amené à cette conception de «radical composé»; il propose ailleurs (p. 198) les noms de radical hydro-carbonique et carbone-hydrique. En choisissant, pour être le premier à être représenté, le nom d'«un des principes des plus abondamment répandus dans la nature», Lavoisier donnait à cette originale «méthode de nommer» les plus grandes chances de se répandre à travers toute la nomenclature. Non seulement Hydr(o)- sera repris dans de très nombreux mots: hydrocarbure (1809; en anglais, 1807), hydracide (1814), dans le morphème suffixé -hydrique proposé par Berzelius pour les hydracides (chlorhydrique, sulfhydrique, etc.), mais beaucoup d'autres «représentants» vont être imaginés par les chimistes.

Il serait fastidieux de les citer tous, il suffira d'indiquer les plus connus ou les plus notables. Ald- représente aldéhyde dans aldol, aldose; am-, ammoniaque dans amide, amine ; ar-, aromatique dans aryle ; az (o)- azote, dans azö̈que, hydrazine ; but-, butyrique dans butyle, et butyle dans butane; eth-, éther dans éthyle et éthane; méth-, méthyle dans méthane et méthacrylique; phtal-, naphtaléne dans phtalique; prop-, propionique dans propane; quin-, quinique ou quinine dans quinoléine; ribo-, ribonique et ribose dans ribonucléique; stér- stérol dans stéride et testostérone; val-, valérique dans valine, etc. Tout le vocabulaire savant suivra l'exemple des chimistes: physique, sciences naturelles, médecine ne peuvent se passer de ces «représentants»: leucocyte devient leuc- ou leukdans leucémie et interleukine; protoplasme devient -plasme dans cytoplasme et ergatoplasme; radiographie se réduit à -graphie dans artériographie et tomographie; stratosphère à strato-dans stratopause; sclérotique à sclér(o) dans sclérotomie et scléral; télé-, dans télécabine et téléski représente téléphérique. Le vocabulaire courant n'échappe pas à ce système: tout le monde sait que les pétrodollars ont leur source dans le pétrole et non dans la pierre.

Il est certain que cette manipulation des formes linguistiques obéit au principe d'économie qui s'impose à tout langage. Le mot, cité plus haut, de testostérone, rassem- 
ble testicule, stérol et hormone, évitant les interminables dénominations périphrastiques que condamnaient les nomenclateurs de 1787. Mais il faut reconnaître que cela ne va pas sans créer un certain nombre d'ambiguiités. Pour reprendre l'exemple de $h y d r(o)-$, on peut noter d'abord un emprunt ancien au grec $\delta \delta \rho(0)$, de $v \delta \omega \rho$ «eau», par ex. dans hydrophobie ou hydrostatique, repris par Lavoisier dans la formation de hydrogène, avec un emploi spécial «liquide organique» dans un mot comme hydrocèle. Dans une seconde série de mots, hydr(o) représente hydrogène, selon l'exemple donné par Lavoisier. Mais les chimistes utilisent concuremment le premier hydr(o)- (ex. hydrate et hydrosilicate) et le second (ex. hydrocarbure et hydracide). De plus un troisième hydr(o)-, du nom d'un cnidaire nommé hydra par Linné (par analogie avec la mythologique hydre de Lerne) est à l'origine de mots comme hydraires, hydrozoaires et hydrocoralliaires. Si les dictionnaires ne renvoient pas à 1 ou 2 ou 3 hydr $(o)$ - pour préciser l'étymologie des mots ainsi préfixés, ils n'apportent aucune information sérieuse. Il ne suffit pas de relever, comme le TLF ou le Nouveau Petit Robert, sous hydr(o)-, un sens «eau» et un sens «hydrogène», il faut encore préciser lequel de ces deux sens est perceptible dans le mot construit. En tout cas, aucun des deux ne permet d'interpréter hydrozoaires et hydrocoralliaires, qui n'ont de rapport qu'avec hydre.

D'ailleurs le même genre d'ambiguïté est à craindre avec les suffixes. Par exemple, le suffixe -ite n'a rien d'univoque dans le vocabulaire savant. Le définir tout simplement comme servant à désigner les maladies de caractère inflammatoire (bronchite) ne rend compte que d'un de ses emplois: on ne peut l'invoquer pour expliquer des mots comme sulfite ou leucite. Que des sciences aussi différentes que la médecine et la minéralogie utilisent des suffixes homophones, mais qui sont autant de morphèmes distincts, cela ne crée pas un vrai problème; mais il n'en est pas de même si le fait se produit à l'intérieur d'une même science. Les chimistes n'ont pas respecté scrupuleusement le modèle de 1787; s'ils ont essayé d'endiguer, on l'a vu, le flot des suffixés en -ine, ils ont accepté de faire servir le suffixe -one à la désignation de corps chimiques de nature différente; il en est de même pour -ane.

Quand des analyses de plus en plus fines, des instruments et des techniques de pointe toujours plus efficaces font découvrir presque chaque année des combinaisons ou des molécules nouvelles, le temps semble manquer pour rester inflexiblement attaché à l'idéal condillacien d'une «langue bien faite». Cependant, sous l'empire de l'utopie directrice de 1787 , la chimie est sans doute, de toutes les sciences, celle qui a élaboré la langue philosophiquement la meilleure; après, bien sûr, celle des mathématiques, mais celle-ci a mis plus de deux mille ans à se chercher !

\section{RÉFÉRENCES}

LAVOISIER (1789): Traité élémentaire de chimie, Mémoires, tableaux et Synonymie de 1787 réunis dans le $3^{\mathrm{e}}$ tome, Nouvelle édition, Cuchet.

Annales de Chimie et Physique, Paris, 1789 et suiv.

THOMSON (1807) : Système de chimie, trad. fr. Paris, 1809, Introduction de Berthollet.

THÉNARD (1824) : Traité de chimie, $4^{\mathrm{e}}$ éd, Paris.

Académie des Sciences, Comptes rendus (depuis 1782).

Bulletin, puis Journal de Pharmacie (1809-1816).

FOURCROY (1806): Philosophie chimique, $3^{\mathrm{e}}$ éd., Paris.

CUVIER (1837-1838): Histoire des progrès des sciences naturelles depuis 1789, éd. de Bruxelles.

CONDILLAC (1780): Logique, t. XXII des CEuvres complètes, Paris, (1798). 